\title{
The Next-Generation Holter Revolution: from Analyse-Edit-Print to Analyse-Print
}

\author{
J Pardey, S Jouravleva \\ Oxford Instruments Medical Ltd, United Kingdom
}

\begin{abstract}
A new three-channel Holter analysis algorithm called Medilog ADAPT is presented and its modular design is described. The use of three-channel analysis improves the accuracy and robustness of the algorithm beyond that of two-channel algorithms and supports the direct printing of reports from unedited analysis results. It also enables Medilog ADAPT to continue working even during periods when two channels are unusable due to signal drop-out or noise corruption. Beat classification uses neural network estimators of Bayesian a posteriori probabilities. A new Holter system running Medilog ADAPT has been performance tested according to ANSI/AAMI standards EC38:1998 and EC57:1998. Gross statistics for QRS Se, $Q R S+P, V E B$ Se and $V E B+P$ are $99.9 \%, 99.9 \%, 98.4 \%$ and $97.6 \%$ respectively for the 78 non-paced ECGS in the AHA database and 99.9\%, 99.9\%, 97.5\% and 93.6\% respectively for the 44 non-paced ECGs in the MIT-BIH database.
\end{abstract}

\section{Introduction}

When an electrocardiogram (ECG) is analysed on a Holter system the user has to spend time editing the analysis results to correct the errors that the system has made before a report can be printed. These errors occur because the ECG is noisy and the algorithms used in commercial Holter systems have not changed greatly since the pioneering days of Holter in the 1970s. This is despite the fact that these same three decades have seen major advances in signal processing and pattern recognition algorithms such as artificial neural networks. For example, the error back-propagation algorithm that is required to train the multilayer perceptron (MLP) was not invented until the 1980s [1] although the perceptron itself was invented back in the 1940s [2]. Academic researchers have been applying new techniques to Holter analysis as soon as each technique is developed and there is now a large body of literature to substantiate this. But Holter companies have been hesitant to employ these new techniques and as a result most Holter systems still rely on correlation-based template matching and heuristic decision rules. There is therefore a significant technology gap between commercial Holter systems and the advanced algorithms of academe. The objective of the project described in this paper is to close this gap and thereby reduce the number of errors that an analysis algorithm makes to a level where no editing of its results is required prior to printing an analysis report. To achieve this a new three-channel, patient-adaptive, Holter analysis algorithm called Medilog ADAPT has been developed. The use of a third channel significantly increases the accuracy and robustness of the algorithm, and enables it to continue working even during periods when two channels are unusable due to signal drop-out or noise corruption. In the remainder of this paper the design and implementation of Medilog ADAPT is described and some performance results are reported.

\section{Methods}

Medilog ADAPT employs a modular design which allows new modules to be added or an existing module to be unplugged and a new version of it plugged in. The five core modules used in Medilog ADAPT are listed below:

- Resample, an ECG resampling module.

- Filter, an ECG filtering module.

- FindQRS, a QRS detection module.

- FindVEB, a VEB detection module.

- ResolveConflicts, a conflict resolution module.

The first three modules comprise a pre-processing stage, while the fourth module is a feature extraction and classification stage, and the fifth is a post-processing stage. The first four modules are used on each channel individually, as shown in Figure 1 overleaf. Each module is described separately in the rest of this section.

The inputs to the ECG resampling module, Resample, are the channels of raw data stored on a Holter recorder. All sample rates of $125 \mathrm{~Hz}$ or greater are supported, as is the use of a different sample rate on each channel. The Resample module upsamples or downsamples each channel to Medilog ADAPT's internal analysis rate of $250 \mathrm{~Hz}$. The use of a fixed analysis rate means the digital filters in the Filter module do not need to be generated on the fly. This allowed the design and implementation of these filters to be carefully optimised during the development of Medilog ADAPT, as described below. 


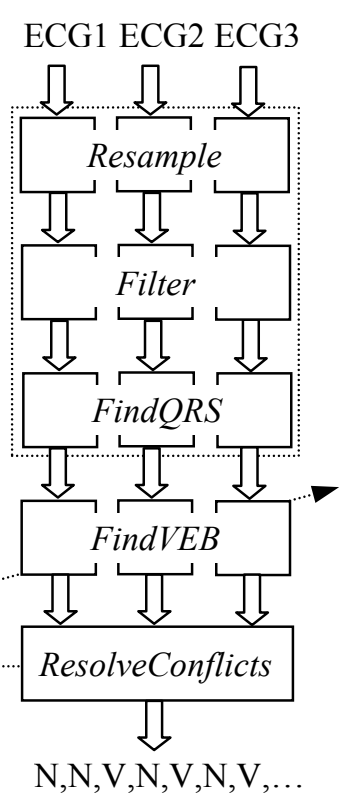

Figure 1. Medilog ADAPT's five core modules.

The inputs to the ECG filtering module, Filter, are the ECG channels from the Resample module. The Filter module performs a number of filtering operations on each channel, including pacemaker spike removal if required. Each channel is bandpass filtered to eliminate or reduce the effects of mains interference, ECG baseline wander, low frequency movement artefact, and high frequency electromyogram noise from non-cardiac muscle. The bandpass filter comprises a finite impulse response (FIR) lowpass filter in series with an infinite impulse response (IIR) highpass filter. The lowpass filter has a passband cut-off frequency of $20 \mathrm{~Hz}$, a stopband cut-off frequency of $40 \mathrm{~Hz}$, a passband ripple of $1 \%$ and a maximum stopband gain of $0.003(-50 \mathrm{~dB})$. The highpass filter has a stopband cut-off frequency of $0.4 \mathrm{~Hz}$, a passband cut-off frequency of $0.5 \mathrm{~Hz}$, a passband ripple of $1 \%$ and a maximum stopband gain of $0.006(-45 \mathrm{~dB})$. To cancel out the nonlinear phase distortion introduced by the IIR filter the input data are IIR filtered, time reversed, and then IIR filtered again $[3,4]$. This bidirectional filtering technique has zero phase distortion and double the filter order, which halves the maximum stopband gain from 0.006 to $0.003(-50 \mathrm{~dB})$. The IIR filter is implemented as cascaded Direct Form 2 biquad sections [5]. The Filter module also generates the first derivative of each filtered ECG channel for subsequent use by the FindQRS module.

The inputs to the QRS detection module, Find $Q R S$, are the channels of differentiated ECG data from the Filter module. The FindQRS module scans each channel using an adaptive slope threshold to detect each QRS complex and its fiducial point [6]. Further low frequency artefact rejection is also performed at this stage. Each QRS detection is followed by a $175 \mathrm{~ms}$ refractory period, and search-back is used to ensure that QRS complexes with rates of change below the slope threshold on the first pass are detected using a lower slope threshold on the second pass. The search-back concept is illustrated in Figure 2.

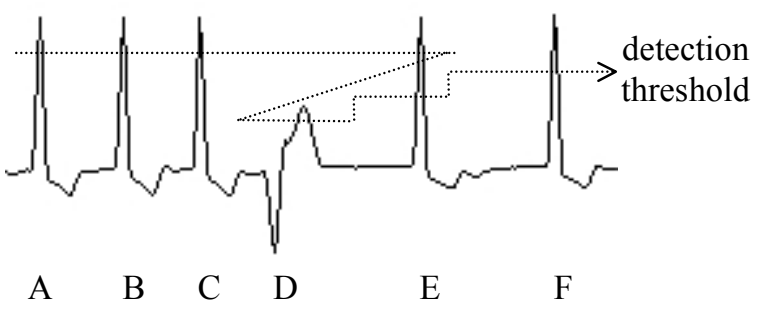

Figure 2. Search-back detects the QRS complexes in the order A, B, C, E, D, F. An adaptive amplitude threshold is shown for illustrative purposes.

The inputs to the VEB detection module, FindVEB, are the bandpass filtered ECG channels from the Filter module plus each channel's QRS fiducial points from the FindQRS module. The FindVEB module first performs feature extraction and normalisation for each detected QRS complex on each channel. Some standard features such as QRS width are used, but other features are novel in that previously they have only been applied to the analysis of electroencephalograms. The amounts of low and high frequency noise in the vicinity of each QRS complex are also estimated at this stage. A look-up table is then used to convert each feature, $x_{i}$, into a probability, $\mathrm{p}\left(\mathrm{N} \mid \mathrm{x}_{\mathrm{i}}\right)$. This is the probability that the QRS complex is supraventricular given the feature, $\mathrm{x}_{\mathrm{i}}$. The probabilities corresponding to all the extracted features are then propagated through an MLP neural network to generate a single output probability, $\mathrm{p}\left(\mathrm{N} \mid \mathrm{x}_{1}, \mathrm{x}_{2}, \ldots, \mathrm{x}_{\mathrm{n}}\right)$. This is the $a$ posteriori probability that the QRS complex is supraventricular given the input probabilities, $\mathrm{p}\left(\mathrm{N} \mid \mathrm{x}_{1}\right)$, $\mathrm{p}\left(\mathrm{N} \mid \mathrm{x}_{2}\right), \ldots, \mathrm{p}\left(\mathrm{N} \mid \mathrm{x}_{\mathrm{n}}\right)$. If the a posteriori probability is above a pre-defined decision threshold the QRS complex is classified as supraventricular, N, otherwise it is classified as ventricular, V. Each channel has its own MLP to ensure that beat classifications on each channel are independent of beat classifications on any other channel.

The MLP weight sets were determined during the development of Medilog ADAPT and no further training is required prior to analysing a recording. Training was by means of the error back-propagation algorithm using a 
learning rate, $\eta$, of 0.01 , a momentum term, $\mu$, of 0.6 and softmax output normalisation [7]. Balanced training and cross-validation sets were used to ensure equal a priori probabilities. The a posteriori probability estimated by the MLP on each channel is given by Bayes' theorem:

$$
\mathrm{p}\left(\mathrm{C}_{\mathrm{k}} \mid \underline{\mathrm{x}}\right)=\frac{\mathrm{p}\left(\underline{\mathrm{x}} \mid \mathrm{C}_{\mathrm{k}}\right) \mathrm{p}\left(\mathrm{C}_{\mathrm{k}}\right)}{\sum_{\mathrm{i}=1}^{\mathrm{K}} \mathrm{p}\left(\underline{\mathrm{x}} \mid \mathrm{C}_{\mathrm{i}}\right) \mathrm{p}\left(\mathrm{C}_{\mathrm{i}}\right)}
$$

where $\underline{x}$ is the feature vector $x_{1}, x_{2}, \ldots, x_{n}, p\left(\underline{x} \mid C_{k}\right)$ is the class-conditional probability of the feature vector $\underline{x}$ given class $\mathrm{C}_{\mathrm{k}}, \mathrm{p}\left(\mathrm{C}_{\mathrm{k}}\right)$ is the a priori probability of class $\mathrm{k}$, and $\mathrm{K}$ is the total number of classes. The formal proof that MLPs can be trained to estimate Bayesian a posteriori probabilities has been published elsewhere [8].

The inputs to the conflict resolution module, ResolveConflicts, are the beat classifications on each channel from the FindVEB module plus the a posteriori probabilities, a subset of the extracted features, and the low and high frequency noise estimates. The ResolveConflicts module employs a number of conflict resolution strategies to (a) accept or reject a beat that has been detected on only a subset of the channels and/or (b) arrive at a final beat classification when there is disagreement between the beat classifications on each channel. The three-channel conflict resolution strategies used in the ResolveConflicts module are similar to published two-channel strategies [9]. However, a significant innovation in Medilog ADAPT is the use of a proprietary algorithm to switch channel priorities dynamically. The channel priorities are then used to rank the reliability of the analysis on each channel and thus ensure that analysis results on good (i.e. higher priority) channels are not confused by conflicting results from poor (i.e. lower priority) channels.

Depending on the final beat classification provided by the ResolveConflicts module the normalisation parameters and MLP weights in the FindVEB module may be updated on one or more channels. This feedback strategy enables the FindVEB module to track changes in QRS morphology over the duration of a recording and can significantly increase its accuracy. However, the use of feedback in Holter algorithms means that misclassified beats may cause the normalisation parameters and MLP weights to be updated in a way that leads to subsequent beats also being misclassified. If this happens then more and more beats may be misclassified in a selfperpetuating cycle. This is equivalent to the problem of positive feedback and system instability in control theory. The risk of instability increases with the duration of the recording and thus becomes particularly relevant in longterm Holter recordings which may extend well beyond 24 hours. Therefore another innovation in Medilog ADAPT is the development of a stable feedback strategy. This has been carefully designed to ensure that if a beat is misclassified on one or two channels the normalisation parameters and MLP weights on those channels are updated so as to decrease the likelihood of subsequent misclassifications. This is equivalent to the use of negative feedback in control systems theory and ensures that errors are self-correcting and not self-perpetuating.

\section{Results}

A typical situation where Medilog ADAPT outperforms two-channel analysis algorithms is shown in Figure 3. Medilog ADAPT has recognised that the top and bottom channels have become unusable but continues to analyse the recording, without interruption or error, using the middle channel.

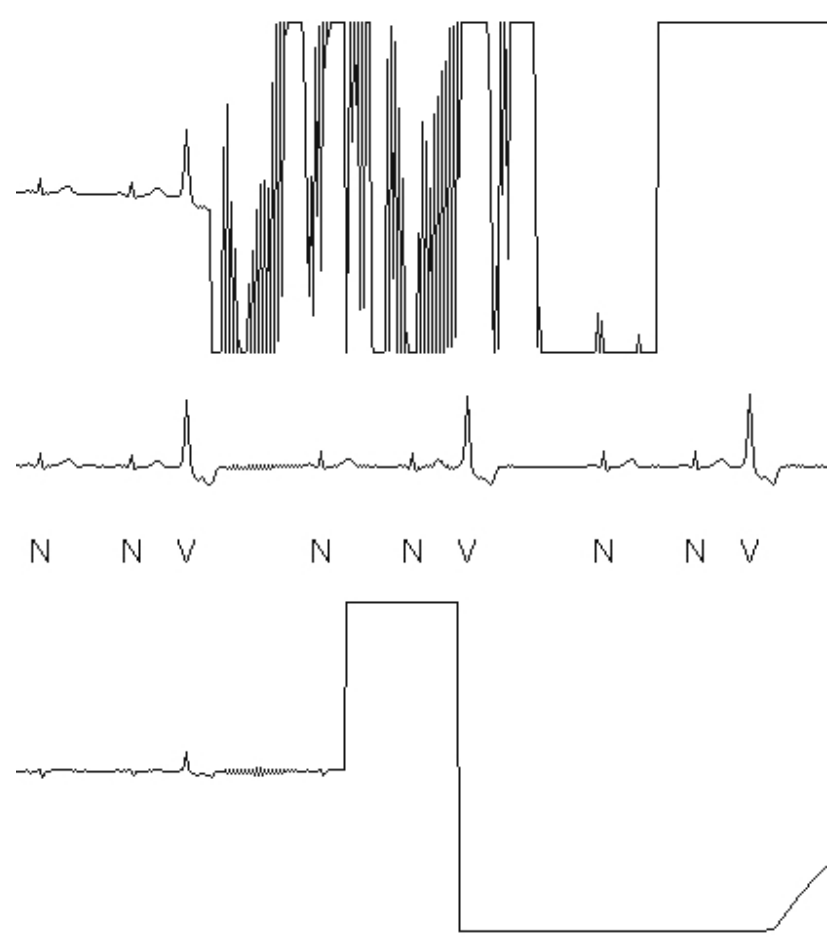

Figure 3. Three-channel analysis using Medilog ADAPT.

The performance of a new Holter system running Medilog ADAPT has been evaluated on the 78 non-paced ECGs in the AHA database and the 44 non-paced ECGs in the MIT-BIH database. The tests were carried out in accordance with ANSI/AAMI standards EC38:1998 [10] and EC57:1998 [11]. The gross statistics for QRS and VEB detection sensitivity (Se) and positive predictivity 
$(+\mathrm{P})$ are given as percentages in Tables 1 and 2, along with published results for the Spacelabs Multiview algorithm [12], the Agilent ST/AR algorithm [13] and the Mortara Veritas algorithm [14]. The results show that Medilog ADAPT generally outperforms the other Holter algorithms, even though the short (thirty-minute) twochannel ECGs in the AHA and MIT-BIH databases do not test the full power of Medilog ADAPT's threechannel analysis algorithm as described in this paper.

Table 1. Performance on the AHA database

\begin{tabular}{|l|cccc|}
\hline & QRS Se & QRS +P & VEB Se & VEB +P \\
\hline Multiview & 99.76 & 99.87 & 95.44 & 96.99 \\
ST/AR & 99.80 & 99.87 & 95.96 & 98.34 \\
Veritas & 99.88 & 99.89 & 94.07 & 97.72 \\
\hline ADAPT & 99.90 & 99.90 & 98.42 & 97.62 \\
\hline
\end{tabular}

Table 2. Performance on the MIT-BIH database

\begin{tabular}{|l|cccc|}
\hline & QRS Se & QRS +P & VEB Se & VEB +P \\
\hline Multiview & 99.82 & 99.85 & 93.00 & 90.05 \\
ST/AR & 99.66 & 99.86 & 94.25 & 96.38 \\
Veritas & 99.93 & 99.85 & 95.44 & 96.90 \\
\hline ADAPT & 99.87 & 99.91 & 97.46 & 93.60 \\
\hline
\end{tabular}

The important point to note in Tables 1 and 2 is that Medilog ADAPT has significantly higher VEB sensitivity than the other Holter algorithms at the expense of slightly lower VEB positive predictivity. This reflects the fact that it is more important to detect VEBs and hence ventricular arrhythmias than to reject a few false positive VEBs during periods of heavily noise-corrupted ECG. Enhanced noise rejection in the next release of Medilog ADAPT will increase VEB positive predictivity, and thus further support users in a move away from the editing of analysis results and towards the direct printing of analysis reports.

\section{Discussion and conclusions}

A new three-channel Holter analysis algorithm called Medilog ADAPT has been presented and its modular design described. The use of three-channel analysis improves the accuracy and robustness of the algorithm beyond that of two-channel algorithms and supports the direct printing of reports from unedited analysis results. It also enables Medilog ADAPT to continue working during periods when two channels are unusable due to signal drop-out or noise corruption. Medilog ADAPT typically takes about 75 seconds to analyse a 24-hour three-channel ECG on a $1.8 \mathrm{GHz}$ PC running Microsoft Windows XP.

\section{Acknowledgements}

The first author would like to thank Professor Lionel Tarassenko at Oxford University whose discussions over lunch at St John's College gave rise to the project in its earliest form.

\section{References}

[1] Rumelhart DE, Hinton GE, Williams RJ. Learning representations by back-propagating errors. Nature 1986; 323:533-6.

[2] McCulloch WS, Pitts W. A logical calculus of the ideas immanent in nervous activity. Bulletin of Mathematical Biophysics 1943; 5:115-33.

[3] Longini RL, Giolma JP, Wall C, Quick R. Filtering without phase shift. IEEE Trans Biomed Eng 1975; 22:432-3.

[4] Gustafsson F. Determining the initial states in forwardbackward filtering. IEEE Trans Signal Processing 1996; 44:988-92.

[5] Smith SW. The Scientist and Engineer's Guide to Digital Signal Processing. California Technical Publishing, 1997.

[6] Engelse WAH, Zeelenberg C. A single scan algorithm for QRS detection and feature extraction. IEEE Computers in Cardiology 1979; 6:37-42.

[7] Bishop CM. Neural Networks for Pattern Recognition. Oxford University Press, 1995.

[8] Richard MD, Lippmann RP. Neural network classifiers estimate Bayesian a posteriori probabilities. Neural Computation 1991; 3:461-83.

[9] Forbes AD, Helfenbein ED, Heumann JM, Jimison HB, Lindauer JM, Platt JS. Ambulatory arrhythmia analysis - a dual channel Bayesian approach. IEEE Computers in Cardiology 1985; 12:373-6.

[10] ANSI/AAMI EC38:1998 Ambulatory Electrocardiographs.

[11] ANSI/AAMI EC57:1998 Testing and Reporting Performance Results of Cardiac Rhythm and ST Segment Measurement Algorithms.

[12] Spacelabs Medical. Arrhythmia system performance evaluation guidelines. Spacelabs Clinical Bulletin Series, 1996; 6(11).

[13] Agilent Technologies. Assessing ST/AR arrhythmia performance. Application Note 5980-1199E, 2000.

[14] Mortara Instruments. Arrhythmia algorithm performance analysis.

www.mortara.com/OEM_arrhythmiadetect_specs.htm

Address for correspondence.

Dr. J. Pardey.

Oxford Instruments Medical Ltd, Manor Way, Old Woking,

Surrey GU22 9JU, United Kingdom

E-mail: james.pardey@oxinst.co.uk 